\title{
Effects of Radiation on Protein
}

\author{
Rong Ke Zhang ${ }^{1,2}$, Jia Hua Lyu², Tao Li ${ }^{1,2}$ \\ ${ }^{1}$ School of Clinical Medicine, Southwest Medical University, Luzhou 646000, Sichuan, China, ${ }^{2}$ Department of Radiotherapy, Sichuan \\ Cancer Hospital \& Institute, School of Clinical Medicine, University of Electronic Science and Technology of China, Chengdu \\ 610041, Sichuan, China
}

\begin{abstract}
Radiotherapy is one of the most common treatments used for solid tumors. Proteins are important components of all cells and tissues, and the main material basis of life. In the present research, more attention has been paid to the relationship between tumor radiotherapy and glycolipid metabolism, but less attention has been paid to the post-radiation effect of proteins. This article discusses the direct and indirect effects of radiation on proteins. The direct effects include changes in the structure and function of proteins, as well as to their synthesis and degradation, with the most specific effects observed on the post-translational modification of proteins. Indirect effects include changes in the whole-body nutrition level of patients, including the process of protein intake, digestion, absorption and metabolism. This article also addresses the impact of advanced radiotherapy technology, such as proton therapy and heavy ion therapy on proteins, and emphasizes the importance and feasibility of protein-supplying nutritional treatment during the "peri-radiotherapy period".
\end{abstract}

Key words: Cancer; Radiotherapy; Protein; Nutrition therapy

\section{Introduction}

Radiation therapy is one of the most important comprehensive treatments for malignant tumors. Approximately $50 \% \sim 70 \%$ of patients need to receive radiation therapy at some point during the treatment process [1]. However, radiation inevitably affects various proteins in the human body. There are many different types of proteins in the human body, which are involved in all life processes ranging from growth and development to immunity, recovery from disease or injury, normal homeostasis and reproduction and heredity. The effects of radiation on proteins are reflected in all levels of the body, including the organs, tissues, cells and molecules. Macroscopically, the loss of body protein will directly lead to malnutrition in cancer patients, which will affect the accuracy of radiotherapy, lead to increased adverse reactions to radiotherapy and reduce the tolerance of radiotherapy. Radiotherapy also has an impact on protein structure and metabolism, and the precise effects depend on the radiosensitivity of different tissues.

As is shown in Figure 1, radiation has both direct and indirect effects on proteins. Direct effects refer to the direct damage caused by radiation to all structures or tissues containing proteins in the body, while indirect effects (at the whole body level) refer to the whole process of radiation effecting proteins from outside into the body. This article reviews the various effects of tumor radiotherapy on

Corresponding author: Tao Li, MD, PhD, Department of Radiotherapy, Sichuan Cancer Hospital \& Institute, School of Clinical Medicine, University of Electronic Science and Technology of China, Chengdu 610041, Sichuan, China; Tel:+86 289542 0173; Fax: +86 288542 0590; Email: litaoxmf@126.com proteins, and provides a multi-dimensional reference for scientific research and clinical treatment.

\section{Direct Effects of Radiation on Proteins \\ Effects of radiation therapy on the structure and function of proteins}

Proteins are chains of amino acids linked by peptide bonds. Proteins have four structural levels (primary through quaternary). The primary structure refers to the sequences of all amino acid residues in the polypeptide chain. The secondary structure is the local spatial conformation of the main chain, which mainly includes $\alpha$-helices and $\beta$-sheets. The tertiary structure is a more complex structure formed on the basis of the secondary structure, which involves the relative spatial position of all amino acid residues in the peptide chain. The quaternary structure is the spatial conformation formed by each subunit of proteins. The functions of a protein are closely related to its complex molecular structure.

Radiation can destroy the molecular structure of proteins at several levels. Interestingly, amino acid residues of the same type may have different radiation sensitivities based on their position (active/inactive center) within the protein molecule. The effects of radiation on the primary structure of a protein include peptide chain ionization, peptide bond cleavage, thiol hydrogenation, reduction of disulfide bonds, and oxidation of side hydroxyl groups. The thiol (-SH) group of cysteine is important to the enzyme's active center. Under hypoxic conditions, ionizing radiation leads to a decrease in the amount of cysteine in the primary structure of RNAse, greatly reducing the enzyme's activity. In addition, the higher-level structure of many proteins also changes after being exposed to radiation. For example, in 
the absence of oxygen, bovine serum albumin coagulates and is denatured after irradiation, and the ratio of molecular aggregates increases with dose of X-ray irradiation. The changes in the protein structure that occur after ionizing radiation are closely related to the individual protein's content of specific amino acids, the presence or absence of oxygen at the time of irradiation, and the irradiation dose [2].

\section{Effects of radiation therapy on the synthesis and degradation of proteins}

\section{Effects of radiation therapy on muscle proteins}

A common side effect of radiotherapy for head and neck cancer is radiation-induced myopathy, which sometimes seriously affects the patient quality of life. In a study of neck muscle atrophy and soft tissue fibrosis after lymph node dissection and radiotherapy for oral cancer, Kim J et al. [3] found that the left sternocleidomastoid muscle (lesion side) of the patient was atrophied more significantly with the passage of time after radiotherapy. Various indicators of muscle tissue damage after radiotherapy can be seen under a microscope, including the following: 1) epithelial-like muscle cells can be seen as round, highchroma cell nuclei clustered in the muscle cytoplasm; 2) some cells contain multiple nuclei; 3) extensive dense fibrosis and inflammatory cell infiltration can be seen in the interstitial space; 4) the arteriole or arteriolar wall are greatly thickened, while a few blood vessels dilate. Some studies have suggested that there are three major mechanisms of muscle injury after radiotherapy, that is, damage to muscle precursor cells, damage to muscle blood vessels, and issues with protein metabolism in muscle cells [4]. For the first mechanism, high doses of radiation may affect the proliferation of muscle precursor cells, which inhibits muscle regeneration. For the second, highdose radiation causes vascular damage, leading to delayed muscle ischemia and muscle atrophy. Finally, radiation also has a negative influence on muscle protein metabolism, which means that there is reduced anabolic metabolism and enhanced catabolic metabolism.

Han X et al. conducted an experiment on mice to investigate the early effects of radiation on the laryngeal muscles. After 25 Gy neck irradiation, it was observed that the size of the muscle fibers and myosin in the thyroid arytenoid muscle of C57BL/6J mice were significantly decreased and the level of MuRF1 protein (muscle-specific ring finger protein 1) was remarkably increased. The main cause of muscle atrophy after irradiation is a loss of myosin heavy chains. The sensitivity of myosin heavy chains to radiation varies among subtypes; the loss of IIB myosin is more severe than the loss of IIA/X myosin. The type IIB glycolytic myofibers are susceptible to radiation-induced changes in myofiber size regardless of the total dose of

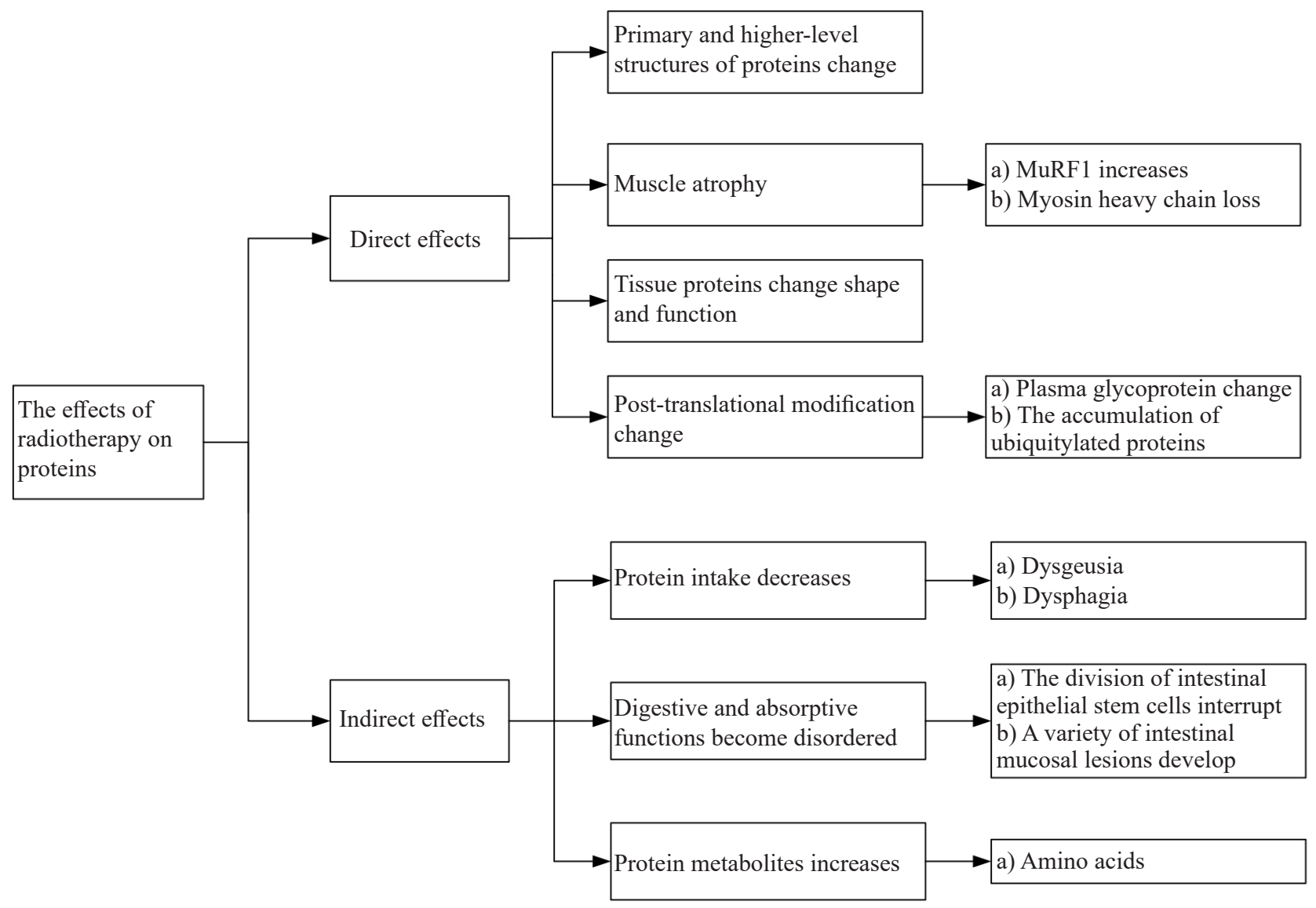

Figure 1 Effects of radiotherapy on protein. 
radiation. In addition, a study found that radiation-induced acute atrophy of muscle fibers and myosin loss were positively correlated with increased MuRF1 expression, suggesting that MuRF1 plays an important role in muscle protein degradation. This is logical because MuRF1 is a muscle-specific E3 ubiquitin ligase that is involved in the activation of the ATP-dependent ubiquitin-proteasome pathway $[5,6]$.

Kim JP et al. demonstrated that the radiosensitivity of myosin heavy chain (MyHC) subtypes in the laryngeal muscles of mice is different, with MyHC IIa being the most sensitive to radiation. The expression of almost all MyHC subtypes was reduced following exposure to radiation, and the expression of MyHC IIa was significantly decreased. The effects on most of the subtypes are time- and dose- dependent. However, following exposure to $20 \mathrm{~Gy}$ irradiation, the expression of almost all MyHC isoforms in muscle gradually decreased over time [7].

In recent years, great progress has been made in radiological technology. By controlling the volume and dose of radiation reaching the muscle, the breakdown of muscle protein can be prevented, reducing muscle atrophy. Since muscle atrophy develops in a dose-dependent manner, cutting the radiation dose to the sternocleidomastoid muscle (SCM) during radiation for head and neck cancer can effectively reduce the occurrence of severe muscle atrophy [8]. Intensity-modulated radiation therapy (IMRT) may make it possible to reduce the dose to the SCM while still delivering high doses to tumor tissue. The V65 (volume of tissue receiving 65 Gy irradiation) is the most sensitive indicator to predict the incidence and extent of muscular atrophy in human patients. To reduce the risk of moderate or severe neck muscle atrophy, Zhang LL et al. suggest imposing a V65 limit of less than $21.47 \%$ to the muscle [9].

\section{Effects of radiation therapy on tissue proteins}

Different tissue proteins exhibit different changes after radiotherapy. Radiation can stimulate the expression of certain proteins in cells, while down-regulating the expression of other proteins. After gamma irradiation, hundreds of proteins were differentially expressed in the blood plasma of rats, among which alpha-2-macroglobulin, chromogranin-a and glutathione peroxidase 3 were dosedependently up-regulated. These three proteins may thus be potential markers for predicting radiation exposure [10].

Graves PR et al. [11] investigated the proteomic changes associated with radiation-induced lung injury, and found that the expression of xanthine dehydrogenase/oxidase, alpha spectrin-2 (fodrin), protein disulfide isomerase, cyclophilin, and annexin II increased in rat lung tissues after radiotherapy. The expression of filamin, alpha-1 proteinase inhibitor III, carbonic anhydrase, glutathione-Stransferase, rho-GDI alpha and peroxiredoxin-2 decreased. Both glutathione-S-transferase and peroxiredoxin-2 are antioxidants that play an important role in protecting lung tissues from oxidative stress.

Meng QY et al. [12] found that the mechanism underlying the excitatory effect of low-dose radiation may be related to the down-regulation of a $24.5 \mathrm{kD}$ protein, but $24.5 \mathrm{kD}$ protein has not been identified. After 4 hours of whole body irradiation with $75 \mathrm{mGy}$, the $24.5 \mathrm{kD}$ protein expression in the thymus cells of mice was significantly lower than that of the control group. At the final concentration of $6.25 \mathrm{mg} / \mathrm{L}$, the loss of this protein component not only inhibits the proliferation of normal splenocytes, but also alleviates $\mathrm{X}$-ray-mediated damage to chromosomes.

Jeung AH used 2D-DIGE technologies to detect changes in proteins in the liver and spleen of irradiated mice. In liver tissue, the expression level of ROS-related proteins, such as cytochrome c, glutathione $\mathrm{S}$ transferase, NADH dehydrogenase and peroxiredoxin VI increased after radiation. In the spleen, the expression level of various apoptosis-related proteins increased, including iodothyronine, CD59A glycoprotein precursor, Fas antigen and tumor necrosis factor-inducible protein TSG-6n precursor. Therefore, the expression of different proteins varies in different tissues after irradiation [13].

\section{Effects of radiotherapy on post-translational modi- fications of proteins}

Post-translational modifications (PTMs) play a vital role in the biological functions of proteins. The PTMs include ubiquitination, phosphorylation, glycosylation, acetylation, and so on. Under the influence of radiation, the PTMs may change.

Glycosylation plays an important role in biological processes such as the immune response and inflammation, and radiation may be associated with protein damage during glycosylation [14]. Toth $\mathrm{E}$ et al. [15] indicate that the glycosylation of human plasma proteins can be altered by radiation and may last for months. In their study, the glycosylation of seven major plasma proteins (alpha1-acid glycoprotein, alpha-2-hs-glycoprotein, alpha-2glycoprotein 1, complement factor $\mathrm{H}$, alpha-globin, kalin 1 , and serum transferrin) was analyzed in 10 patients with neck squamous cell carcinoma (HNSCC). All patients were subjected to IMRT using 6-MV photons. The abundance of 99 glycoforms changed after radiotherapy, with some glycoforms increasing sharply, while others changed in the opposite direction. Due to the small sample size of the study and the large variation in glycosylation among individuals, it remains to be verified whether the results can be used to evaluate the efficacy of radiotherapy and the rehabilitation of patients.

A 2016 study reported the effects of carbon-ion (C-ion) irradiation and X-ray irradiation on ubiquitylated proteins in human SW620 and SW480 colorectal cancer cell lines. Compared with the $\mathrm{X}$-ray irradiated cells, $\mathrm{C}$-ion irradiation more strongly induced the accumulation of ubiquitylated 
proteins (6 Gy irradiation was required for X-rays, while only 3 Gy were required for $\mathrm{C}$-ion radiation to cause damage to SW620 cells). The use of proteasome inhibitors may increase the sensitivity of cells to $\mathrm{C}$-ion radiation. For example, Isozaki $\mathrm{T}$ et al. confirmed that the cytotoxicity of C-ion irradiated SW620 cells was enhanced by treatment with a proteasome inhibitor (epoxymycin). Researchers have observed that some of the ubiquitylated proteins were partially co-localized with $\gamma-\mathrm{H} 2 \mathrm{AX}$ or TP53BP1, indicating that $\mathrm{C}$-ion irradiation induced the ubiquitylation of proteins with DNA repair-related functions [16-19].

\section{Indirect Effects (whole body level)}

The most important effects of radiation therapy on the whole body are the changes in the nutritional status of patients with malignant tumors. Radiation can affect any or all steps in the patient's protein intake, digestion, absorption and metabolism. The extent of the effects and mechanism underlying the effects of radiotherapy on the nutritional status of patients different based on the dose and affected tissue or process (Figure 2).

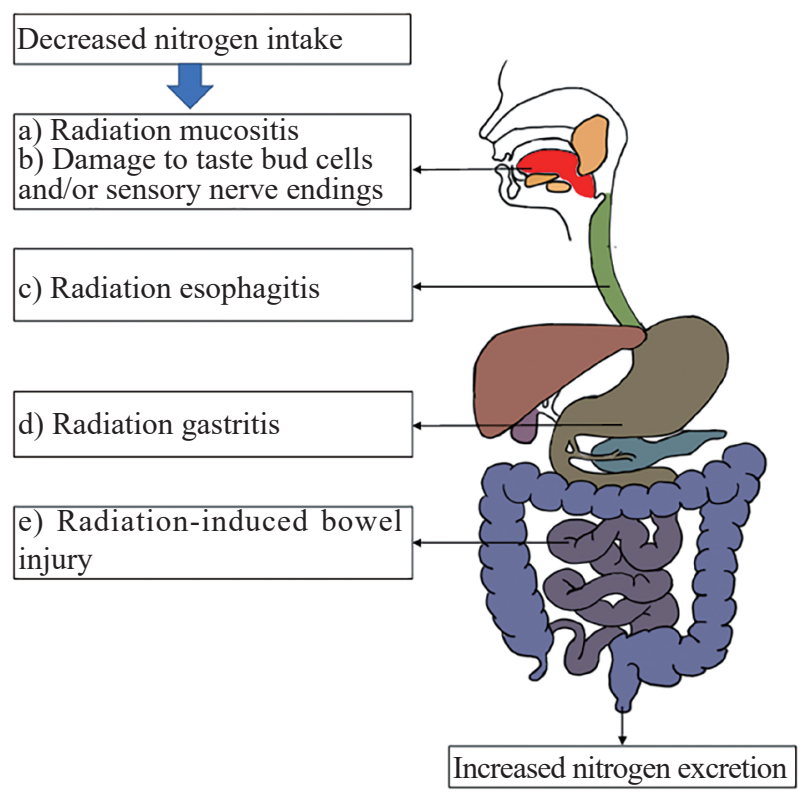

Figure 2 Indirect effects of radiation: how radiation therapy leads to a negative nitrogen balance.

\section{Dietary intake}

During radiotherapy, the patients' dietary intake is typically reduced, and their protein intake decreases along with this overall reduction. One study observed that there was a positive correlation between the decrease in protein intake and the mid-upper arm circumference during the $3 \mathrm{rd}$ week of radiation therapy, but the correlation was weak ( $\mathrm{r}$ $=0.287 ; P=0.024)$. However, the muscle mass gradually decreases and the incidence of cachexia increases with the duration of treatment [20].
Most patients suffer from dysgeusia during radiotherapy. Exposure of the anterior part of the tongue to low-dose irradiation (e.g., 20 Gy) will cause temporary taste loss. Interestingly, exposure to high doses of radiation on the root of the tongue did not cause acute taste loss. Thus, if radiation must be administered, Yamashita $\mathrm{H}$ et al. [21] noted that it was most important to reduce the dose reaching the first third of the tongue in order to protect the sense of taste and increase the dietary intake of patients.

In a comparative study of IMPT versus IMRT for oropharyngeal cancer, the symptoms of dysgeusia, dry mouth, difficulty swallowing/chewing, loss of appetite and fatigue were lower in the IMPT patients than in the IMRT patients. Unlike other radiotherapy technologies, proton therapy has a Bragg peak, which can reduce the dose reaching adjacent healthy tissue, thereby minimizing side effects. Therefore, the use of IMPT in oropharyngeal or head and neck cancer can not only improve the tumor control rate, but also reduce the radiation dose to the taste buds and other oral structures [22,23].

In summary, for patients with malignant tumors receiving head and neck or chest radiotherapy, radiation will destroy taste bud cells and taste sensory nerve endings, affecting the patients' taste and reducing their appetite. In addition, side effects such as radiation-induced oral mucositis and esophagitis directly affect the intake of carbohydrates, fats, proteins, cellulose and other nutrients, resulting in weight loss and dystrophy [24].

\section{Digestion and absorption}

Radiotherapy is one of the most effective methods used to treat pelvic and abdominal malignancies. It has been reported that $35 \% \sim 61 \%$ of patients with pelvic malignancies have received pelvic radiotherapy, while up to $75 \%$ of these patients experience gastrointestinal symptoms of varying degrees [25]. Most abdominal radiotherapy involves gastrointestinal tissue. At 40 50 Gy, 1\% 5\% of patients had reported gastrointestinal side effects. At doses above $65 \mathrm{~Gy}, 25 \% \sim 50 \%$ of the patients developed severe radiation gastroenteritis. Such patients can die from water and electrolyte disorders, protein loss and/or infection [26].

Radiation can cause gastrointestinal mucosal damage, often manifested as abdominal pain, diarrhea, nutritional absorption disorders, and so on. The intestinal epithelium is sensitive to radiation, and radiation can prevent the division of intestinal epithelial stem cells. This disrupts the dynamic balance between proliferating cells and apoptosis in the intestine. Acute radiation enteritis manifests as intestinal wall edema and intestinal mucosal shedding; chronic radiation enteritis presents as severe vasculitis and submucosal fibrosis, and may result in stenosis, ulcers, and fistulas. In such cases, the morphology and function of the intestinal epithelium have been destroyed, and the body is unable to maintain its demand for protein and water $[27,28]$. 


\section{Metabolism}

After being irradiated, the lysosome is destroyed, releasing cathepsin, while the patient's dietary intake is also significantly reduced. The combined effects of these factors results in enhanced catabolism and reduced anabolism of proteins. Therefore, the levels of free amino acids increase, as does gluconeogenesis, leading the body to be in a state of negative nitrogen balance. Li XL et al. [29] suggested that the radiotherapy-related acute response can enhance protein catabolism. That study involving seven cancer patients observed that most patients had a negative nitrogen balance, that is to say, their nitrogen intake was reduced and excretion was increased after radiotherapy. It is important to note that tumor-associated malnutrition is not a simple nutrient deficiency. Due to the uniqueness of protein metabolism in tumor cells, the reprogramming of protein metabolism in the host (tumor patient) causes abnormal amino acid metabolism and changes the plasma amino acid expression.

Protein catabolism in mammals speeds up after irradiation [2]. Among the metabolites of protein, the changes in the amino acid levels are the most obvious. After systemic irradiation with X-rays (6 Gy), the levels of leucine and phenylalanine in the spleens of mice increased $5 \sim 6$ folds within 24 hours. In addition, the excretion of creatine and taurine also increased after radiation exposure. These changes are sometimes used as auxiliary indicators to judge the degree of radiation damage [2].

\section{Nutrition Counseling and Nutritional Therapy}

Based on the above information about the effects of radiation on protein, it can be concluded that radiation can cause malnutrition in patients, mainly protein malnutrition. And malnutrition is not conducive to the smooth progress of radiotherapy. In addition to improving the technology being used for irradiation to reduce muscle and tissue protein damage, nutrition counseling and nutritional therapy should be provided to ensure that there is adequate protein intake and digestion.

Ravasco P et al. [30] evaluated the effects of nutritional supplementation and nutrition counseling in patients with head and neck cancer undergoing radiotherapy, and found that the energy and protein intake increased in the study group, while that in the control group decreased. Therefore, these authors emphasized the importance of dietary counselling during radiotherapy to prevent deterioration of the nutritional status of patients. In another study, nutritional intervention during radiotherapy had a positive effect on the patient outcomes, with benefits similar to or greater than those of counselling. At the same time, a combination of clinical and radiodosimetry factors was also evaluated to better predict the need for prophylactic gastrostomy (PEG) in patients with head and neck cancer, thereby reducing the risk of malnutrition caused by radiation-related dysphagia [31]. An ECOG score $>1$, concurrent chemotherapy, and a higher mean dose to the oropharynx $+1 \mathrm{~cm}$ margin were important factors that determined the need for prophylactic PEG tube insertion during radiotherapy. Kalaiselvan $\mathrm{R}$ et al. [30] suggested that the majority of patients with chronic radiation enteritis require long-term parenteral nutrition (HPN) [32]. The implementation of detailed nutritional management can effectively improve various nutritional indicators of patients with esophageal cancer (receiving concurrent chemoradiotherapy), improve the nutrition knowledge level, and reduce the incidence of adverse reactions to chemoradiotherapy [33].

$\mathrm{Li} \mathrm{T}$ et al. [34] indicated that patients with malignant tumors need to undergo comprehensive nutrition management before, during and after radiotherapy. During radiotherapy, the type of nutritional therapy (enteral/ parenteral nutrition) needs to be recommended based on individual differences. On the basis of achieving the recommended target amount $25 \sim 30 \mathrm{kcal} /(\mathrm{kg} \cdot \mathrm{d})$, the energy requirements of specific patients should also be adjusted according to the tumor load, stress status, and acute radiation injury. A protein intake of $1.2 \sim 1.5 \mathrm{~g} /(\mathrm{kg} \cdot \mathrm{d})$ is generally recommended for all patients, while intake of $1.5 \sim 2.0 \mathrm{~g} /(\mathrm{kg} \cdot \mathrm{d})$ is recommended for patients with severe malnutrition. Patients with cachexia should increase their intake to $2.0 \mathrm{~g} /(\mathrm{kg} \cdot \mathrm{d})$.

\section{Conclusions and Outlook}

There are abnormalities in protein synthesis and catabolism in patients with malignant tumors, and protein breakdown is often faster than synthesis. Some patients develop cachexia. Studies have found that patients have reduced muscle content after radiotherapy. Loss of skeletal muscle and fibrosis of the muscles in the irradiated area will not only cause malnutrition, but also directly affect the motor function of patients. The effects of radiation on proteins need to be further clarified so that appropriate interventions can be developed. In particular, future studies should focus on determining the effects of different doses and different segmentation methods on proteins in different organs and tissues. It is also necessary to investigate whether alternative radiation types, fractionation patterns or radioprotective agents can be used to minimize the adverse effects of radiation of the patient's normal proteins.

\section{Conflict of Interest}

The authors do not have any commercial or financial conflict of interest to disclose.

\section{Funding}

This work was supported by a grant from the Jie Peng Wu Foundation Project (No. 320.6750.18170). 


\section{References}

1. Zhu GY, Wang LH. Radiation oncology. Beijing: People's Medical Publishing House Co., LTD. 2016.

2. Gong SL. Medical Radiobiology. 4 st ed. Beijing: Atomic Energy Press, 2015.

3. Kim J, Shin ES, Kim JE, Yoon SP, Kim YS. Neck muscle atrophy and soft-tissue fibrosis after neck dissection and postoperative radiotherapy for oral cancer. Radiat Oncol J 2015;33(4):344-9.

4. Fajardo L, Berthrong M, Anderson R. Alimentary tract radiation pathology. Oxford: Oxford University Press, 2001.

5. Han X, Pires L, Browne JD, Sullivan CA, Zhao W, Feng X. Increased expression of MuRF1 is associated with radiation-induced laryngeal muscle atrophy. Anticancer Res 2015;35(11):6049-56.

6. Hardee JP, Puppa MJ, Fix DK, Gao S, Hetzler KL, Bateman TA, Carson JA. The effect of radiation dose on mouse skeletal muscle remodeling. Radiol Oncol 2014;48(3):247-56.

7. Kim JP, Khalmuratova R, Jeon SY, Park JJ, Hur DG, Ahn SK, Woo SH, Kang KM, Chai GY. Quantitative analysis of myosin heavy chain expression change in laryngeal muscle after irradiation in rats. Yonsei Med J 2011;52(1):158-64

8. Popovtzer A, Cao Y, Feng FY, Eisbruch A. Anatomical changes in the pharyngeal constrictors after chemoirradiation of head and neck cancer and their dose-effect relationships: MRI-based study. Radiother Oncol 2009;93:510-5.

9. Zhang LL, Wang XJ, Zhou GQ, Tang LL, Lin AH, Ma J, Sun Y. Dose-volume relationships for moderate or severe neck muscle atrophy after intensity-modulated radiotherapy in patients with nasopharyngeal carcinoma. Sci Rep 2015;18(5):18415.

10. Sun JL, Li S, Lu X, Feng JB, Cai TJ, Tian M, Liu QJ. Identification of the differentially expressed protein biomarkers in rat blood plasma in response to gamma irradiation. Int J Radiat Biol 2020;96(6):748-58.

11. Graves PR, Sankala H, Vujaskovic Z. Effect of ionizing radiation on rat lung tissue:A proteomic analysis. Int J Radiat Oncol Bio Phys 2007;69(3):S618.

12. Meng QY, Chen SL, Liu SZ. Expression of proteins in mouse thymocytes induced by low dose X-rays and their biological activity. Chin J Pathophysiol 2003;(07):42-4.

13. Jeung AH, Jin SS. Proteomics analysis of apoptosis-regulating proteins in tissues with different radiosensitivity. J Radiat Res 2006;47(2):147-55.

14. Ma J, Han D, Zhang M, Chen C, Zhang B, Zhang Z, Wang X, Yang S, Guo Y, Okunieff P, Zhang L. Alteration of plasma galactose/ $\mathrm{N}$-acetylgalactosamine level after irradiation. Adv Exp Med Biol 2013;147-53.

15. Toth E, Vekey K, Ozohanics O, Jeko A, Dominczyk I, Widlak P, Drahos L. Changes of protein glycosylation in the course of radiotherapy. J Pharm Biomed Anal 2016;118:380-6.

16. Isozaki T, Fujita M, Yamada $S$, Imadome K, Shoji Y, Takeshi T, Nakayama F, Imai T, Matsubara H. Effects of carbon ion irradiation and $\mathrm{X}$-ray irradiation on the ubiquitylated protein accumulation. Int $\mathrm{J}$ Oncol 2016;49(1):144-52.

17. Jiang YJ, Zang Y. The Roles of 53BP1 in DNA double-strand breaks repair. Chin J Biochem Mol Biol 2016;32(12):1279-85.

18. Micel LN, Tentler JJ, Smith PG, GS Eckhardt. Role of ubiquitin ligases and the proteasome in oncogenesis: novel targets for anticancer therapies. J Clin Oncol 2013;31:1231-8.

19. Endo S, Fujita M, Yamada S, Matsubara H. Fra-1 enhances the radioresistance of colon cancer cells to $\mathrm{X}$-ray or $\mathrm{C}$-ion radiation. Oncol Rep 2018;39:1112-8.

20. Pistoia LF, de Abreu Nunes CH, Andreatta GB, Rabito EI. Dietary intake of cancer patients on radiotherapy. Nutr Hosp 2012;27(6):1936-9. 21. Yamashita H, Nakagawa K, Nakamura N, Abe K, Asakage T, Ohmoto M, Okada S, Matsumoto I, Hosoi Y, Sasano N, Yamakawa S, Ohtomo K. Relation between acute and late irradiation impairment of four basic tastes and irradiated tongue volume in patients with head and neck cancer. Int J Rad Oncol Biol Phys 2006;66(5):1422-9.

22. Sio TT, Lin HK, Shi Q, Gunn GB, Cleeland CS, Lee JJ, Hernandez M, Blanchard P, Thaker NG, Phan J, Rosenthal DI, Garden AS, Morrison WH, Fuller CD, Mendoza TR, Mohan R, Wang XS, Frank SJ. Intensity modulated proton therapy versus intensity modulated photon radiation therapy for oropharyngeal cancer: first comparative results of patientreported outcomes. Int J Radiat Oncol Biol Phys 2016;95(4): 1107-14.

23. Deshpande TS, Blanchard P, Wang L, Foote RL, Zhang X, Frank SJ. Radiation-related alterations of taste function in patients with head and neck cancer: a systematic review. Curr Treat Options Oncol 2018;19(12):72.

24. Li T, Lyu JH, Lang JY, Zhang Z, Jin J, Xu HX, Li W, Cong MH, Zhou FX, Yang DK, Li GW, Kang JB, Shi HP. The enteral nutrition in radiotherapeutic cancer patients. Electron $\mathrm{J}$ Metab Nutr Cance 2017;4(3):272-9.

25. Surgeon Branch of Chinese Medical Doctor Association, Colorectal Surgery Group of Surgery Branch in the Chinese Medical Association. Chinese consensus on diagnosis and treatment of radiation proctitis (2018). Chin J Inflamm Bowel Dis 2019;3(1):5-20.

26. Zhu GY, Luo JW, Yin WB. Radiation oncology. 2nd ed. Beijing: Science and Technology Academic Press, 2006.

27. Ao R, Lu Y. The recent advancement of mechanism research on pathogenesis of radiation proctitis. Prac J Clin Med 2009;6(2):112-4.

28. Rehailia-Blanchard A, He MY, Rancoule C, Vallard A, Espenel S, Nivet A, Magne N, Chargari C. Physiopathology and pharmacological perspectives in the treatment of radiation enteritis. Cancer Radiother 2019;23(3):240-7.

29. Li XL, Zhu ST. Study on nitrogen balance in cancer patients before and after radiotherapy. Acta Nutrimenta Sinica 2004;26(4):319320,322 .

30. Ravasco P, Monteiro Grillo I, Marques Vidal P, Camilo ME. Impact of nutrition on outcome: a prospective randomized controlled trial in patients with head and neck cancer undergoing radiotherapy. Head and Neck 2005;27(8):659-68.

31. Matuschek C, Bolke E, Geigis C, Kammers K, Ganswindt U, Scheckenbach K, Gripp S, Simiantonakis J, Hoffmann TK, Greve J, Gerber PA, Orth K, Roeder H, Hautmann MG, Budach W. Influence of dosimetric and clinical criteria on the requirement of artificial nutrition during radiotherapy of head and neck cancer patients. Radiother Oncol 2016;120(1):28-35.

32. Kalaiselvan R, Theis VS, Dibb M, Teubner A, Anderson ID, Shaffer JL, Carlson GL, Lal S. Radiation enteritis leading to intestinal failure: 
1994 patient-years of experience in a national referral centre. Eur J Clin Nutr 2014;68(2):166-70

33. Zhang HL, Zhang HF, Jiang GF, Lyu JH, Chen Y, Zhang HY. Effects analysis of whole-process nutrition management on enteral nutrition intervention in patients with synchronous radiotherapy and chemotherapy for esophageal cancer. Chongqing Med J 2019;48(9):1594-6.
34. Li T, Lyu JH, Lang JY, Shi HP, Xu HX, Li BS, Zhang Z, Zhu SC, Chen M, Kang JB, Shi AH, Li JC, Zhao C, Liu SX, Yang DK, Li G, Zhou FX, Li H, Dong LH, Cong MH, Shen LF, Zou LJ, Zhao R, Li RQ. Expert consensus on nutririon therapy in cancer patients receiving radiotherapy. Electron J Metab Nutr Cance 2018;5(4):358-65. 